\title{
In Vivo Analysis of Trypanosoma cruzi Persistence Foci at Single-Cell Resolution
}

\author{
Alexander I. Ward, ${ }^{a}$ Michael D. Lewis, ${ }^{a}$ Archie A. Khan, ${ }^{a}$ Conor J. McCann,, Amanda F. Francisco, ${ }^{\text {a }}$ Shiromani Jayawardhana, ${ }^{\text {a }}$ \\ Martin C. Taylor, ${ }^{\text {a }}$ (D) John M. Kelly ${ }^{a}$
}

aDepartment of Infection Biology, London School of Hygiene and Tropical Medicine, London, United Kingdom

bStem Cells and Regenerative Medicine, Great Ormond Street Institute of Child Health, University College London, London, United Kingdom

ABSTRACT Infections with Trypanosoma cruzi are usually lifelong despite generating a strong adaptive immune response. Identifying the sites of parasite persistence is therefore crucial to understanding how T. cruzi avoids immune-mediated destruction. However, this is a major technical challenge, because the parasite burden during chronic infections is extremely low. Here, we describe an integrated approach involving comprehensive tissue processing, ex vivo imaging, and confocal microscopy, which allowed us to visualize infected host cells in murine tissue with exquisite sensitivity. Using bioluminescence-guided tissue sampling, with a detection level of $<20$ parasites, we showed that in the colon, smooth muscle myocytes in the circular muscle layer are the most common infected host cell type. Typically, during chronic infections, the entire colon of a mouse contains only a few hundred parasites, often concentrated in a small number of cells each containing $>200$ parasites, which we term mega-nests. In contrast, during the acute stage, when the total parasite burden is considerably higher and many cells are infected, nests containing $>50$ parasites are rarely found. In $\mathrm{C} 3 \mathrm{H} / \mathrm{HeN}$ mice, but not BALB/c mice, we identified skeletal muscle as a major site of persistence during the chronic stage, with most parasites being found in large mega-nests within the muscle fibers. Finally, we report that parasites are also frequently found in the skin during chronic murine infections, often in multiple infection foci. In addition to being a site of parasite persistence, this anatomical reservoir could play an important role in insectmediated transmission and have implications for drug development.

IMPORTANCE Trypanosoma cruzi causes Chagas disease, the most important parasitic infection in Latin America. Major pathologies include severe damage to the heart and digestive tract, although symptoms do not usually appear until decades after infection. Research has been hampered by the complex nature of the disease and technical difficulties in locating the extremely low number of parasites. Here, using highly sensitive imaging technology, we reveal the sites of parasite persistence during chronic-stage infections of experimental mice at single-cell resolution. We show that parasites are frequently located in smooth muscle cells in the circular muscle layer of the colon and that skeletal muscle cells and the skin can also be important reservoirs. This information provides a framework for investigating how the parasite is able to survive as a lifelong infection, despite a vigorous immune response. It also informs drug development strategies by identifying tissue sites that must be accessed to achieve a curative outcome.

KEYWORDS Trypanosoma cruzi, Chagas disease, chronic persistence, murine imaging, colon, skeletal muscle, skin, chronic, persistence

T he intracellular protozoan parasite Trypanosoma cruzi is the etiological agent of Chagas disease, and it can infect a wide variety of mammalian hosts. Transmission to humans occurs mainly via the hematophagous triatomine insect vector, which deposits infected feces on the skin after a blood meal, with the parasite then intro-
Citation Ward Al, Lewis MD, Khan AA, McCann CJ, Francisco AF, Jayawardhana S, Taylor MC, Kelly JM. 2020. In vivo analysis of Trypanosoma cruzi persistence foci at single-cell resolution. mBio 11:e01242-20. https://doi.org/10.1128/ mBio.01242-20.

Editor Barbara Burleigh, Harvard T. H. Chan School of Public Health

Copyright $\odot 2020$ Ward et al. This is an openaccess article distributed under the terms of the Creative Commons Attribution 4.0 International license.

Address correspondence to John M. Kelly, john.kelly@lshtm.ac.uk.

Received 11 May 2020

Accepted 29 June 2020

Published 4 August 2020 
duced through the bite wound or mucous membranes. Oral, congenital, and blood transfusion are other important transmission routes. Six to seven million people in Latin America are infected with $T$. cruzi (1), and as a result of migration, there are now hundreds of thousands of infected individuals in regions where the disease is not endemic, particularly the United States and Europe $(2,3)$.

In humans, infection normally results in mild symptoms, which can include fever and muscle pain, although in children the outcome can be more serious. Within 6 weeks, this acute phase is usually resolved by a vigorous $\mathrm{CD}^{+} \mathrm{T}$ cell response $(4,5)$, and in most cases, the infection progresses to a lifelong asymptomatic chronic stage, where the parasite burden is extremely low and no apparent pathology is observed. However, in $\sim 30 \%$ of individuals, the infection manifests as a symptomatic chronic condition, although this can take many years to develop. The associated cardiac dysfunction, including dilated cardiomyopathy and heart failure, is a major cause of morbidity and mortality $(6,7)$. In addition, $\sim 10 \%$ of those infected display digestive pathologies, such as megacolon and megaesophagus, which on occasions can occur in parallel with cardiac disease. There is no vaccine against $T$. cruzi infection, and the current frontline drugs, benznidazole and nifurtimox, have limited efficacy, require long treatment regimens, and can have severe side effects $(8,9)$. The global effort to discover new drugs for Chagas disease involves not-for-profit drug development consortia, as well as the academic and commercial sectors $(10,11)$. Progress would benefit considerably from a better understanding of parasite biology and pathogenesis.

One of the major challenges in Chagas disease research is to determine how T. cruzi survives as a lifelong infection, despite eliciting a vigorous immune response which is able to reduce the parasite burden by $>99 \%$. Exhaustion of the parasite-specific CD8 ${ }^{+}$ T cell response does not appear to be the reason (12). Alternative explanations include the possibility that T. cruzi is able to persist in immune-tolerant tissue sites (13) and the potential for the parasite to assume a nondividing dormant form that does not trigger an overt immune response (14). Attempts to investigate these issues in humans have been limited by the long-term and complex nature of the disease and by difficulties in monitoring tissue infection dynamics during the chronic stage. Of necessity, most information on the sites of parasite location in humans has come from autopsy and transplant studies (15), and the relevance of these data to patients in the asymptomatic chronic stage is unclear. Bioluminescence imaging of animal models has therefore been adopted as an approach to explore aspects of host-parasite interaction, pathology, and drug development (16-18). Our previous work and that of others exploited highly sensitive in vivo imaging to monitor mice infected with bioluminescent $T$. cruzi that expresses a red-shifted luciferase (19-21). These experiments showed that mice are useful predictive models for human infections in terms of infection dynamics $(21,22)$, drug sensitivity (23), and the spectrum of cardiac pathology (24). We have also demonstrated that $T$. cruzi infection is pantropic during the acute stage and that the adaptive immune response results in a 100- to 1,000-fold reduction in the whole-animal parasite burden as infections transition to the chronic phase, a process initiated 2 to 3 weeks postinfection. The gastrointestinal (GI) tract, particularly the colon and/or stomach, was found to be a major site of parasite persistence during chronic-stage infections, but it has not so far been possible to identify the infected host cell types in these complex tissues. The immune-mediated restriction to the Gl tract was not absolute, with both host and parasite genetics impacting the extent to which the infection could disseminate to a range of other organs and tissues (22). The severity of chronic cardiac pathology in different mouse strains was associated with the ability of parasites to spread beyond the permissive niche provided by the Gl tract and with the incidence of cardiac infection. This led us to propose a model in which the development of chagasic cardiac pathology was linked with the frequency of the localized inflammatory immune responses stimulated by periodic trafficking of parasites into the heart (13).

More detailed information on the precise sites of parasite survival during chronic infections will provide new insights into pathogenesis and aid the design of both 
immunotherapeutic and chemotherapeutic strategies. The scarcity of parasites during the chronic stage has made addressing this issue a major challenge, with PCR-based approaches being both uninformative with respect to host cell types and unreliable because of the highly focal and dynamic character of infections $(20,23)$. To resolve this, we constructed $T$. cruzi reporter strains engineered to express a fusion protein that is both bioluminescent and fluorescent (25). This allowed individual infected host cells to be visualized routinely within chronically infected mouse tissue. The bioluminescent component facilitates the localization of infection foci within ex vivo tissue samples, and fluorescence then enables histological sections to be rapidly scanned to identify infected cells (26). The utility of this approach has been further extended by using 5-ethynyl-2'-deoxyuridine (EdU) labeling and terminal deoxynucleotidyltransferasemediated dUTP-biotin nick end labeling (TUNEL) assays to explore the replicative status of parasites in situ.

Here, we describe how these enhanced imaging procedures, coupled with modifications to tissue processing, have allowed us to identify the sites of parasite persistence during chronic murine infections. We reveal that the circular muscle layer is the major reservoir of infection in the colon, that skeletal muscle can be an important site of persistence (although this phenomenon appears to be strain specific), and that the skin can harbor multiple infection foci.

\section{RESULTS}

Locating the sites of $T$. cruzi persistence within the external wall of the colon during chronic murine infections. In multiple murine models, with a variety of parasite strains, bioluminescence imaging has revealed that the Gl tract, particularly the large intestine and stomach, is a major site of parasite persistence during chronic $T$. cruzi infection $(20,22)$. However, our understanding of how this impacts pathogenesis has been complicated by the difficulty of precisely locating, and then visualizing, parasite-infected cells. To resolve these technical issues, we infected mice with the $T$. cruzi CL-Luc::Neon line, which constitutively expresses a reporter fusion protein that is both bioluminescent and fluorescent (25), and adapted our dissection procedures to allow a more detailed assessment of parasite location (see Materials and Methods). At various periods postinfection, the colon of each mouse was removed, pinned luminal side up, and peeled into two distinct sections (Fig. 1a and b): (i) the mucosal layers, consisting of thick mucosa, muscularis mucosa, and submucosal tissue, and (ii) the muscular coat, including the longitudinal and circular smooth muscle layers, the enteric neuronal network, and, at the level of the myenteric plexus, intramuscular neurons and extrinsic nerve fibers. The resulting external gut wall mount is thin enough, and sufficiently robust, to allow the full length of the colon to be viewed in its entirety at a 3-dimensional level by confocal laser scanning microscopy. Using this approach, each bioluminescent focus in peeled tissue from chronically infected mice could be correlated with fluorescent parasites in individual infected host cells (Fig. 1c and d). The resulting images revealed that the limit of detection achievable by bioluminescence imaging is less than 20 parasites. This level of sensitivity, in an ex vivo context, confirms the utility of this model for studies on infection dynamics (22) and drug and vaccine efficacy $(24,27,28)$. In infected host cells, the number of parasites could be determined with precision using full-thickness serial z-stacking (Fig. 1e; Fig. S1). This allowed us to establish that the total number of parasites persisting in the external colonic wall (tunica muscularis) of a chronically infected mouse is typically in the range of a few hundred $(697 \pm 217 ; n=16)$, although this number can be higher if the tissue contains one or more "mega-nests" (for an example, see Fig. 1c, highlighted in yellow).

When we compared parasite distribution in the external gut wall during acute and chronic murine infections, the most striking difference was the presence in the chronic stage of some host cells that were infected with $>200$ parasites (Fig. 2). The existence of these mega-nests resulted in a major alteration in parasite number distribution at the level of single infected host cells (Fig. 1; Fig. 2 b to d). In acute infections, parasites were spread among many more host cells, with the average parasite content per 
(a)

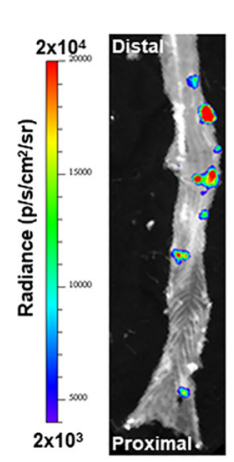

(b)

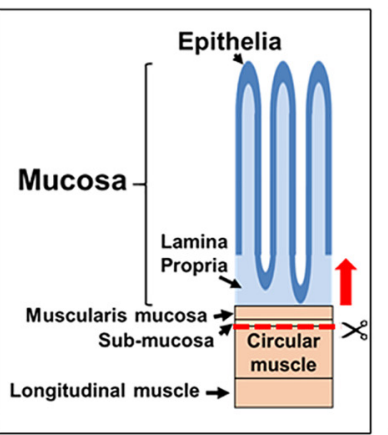

(c)

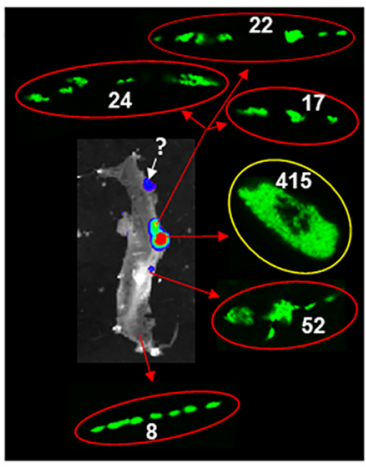

(d)

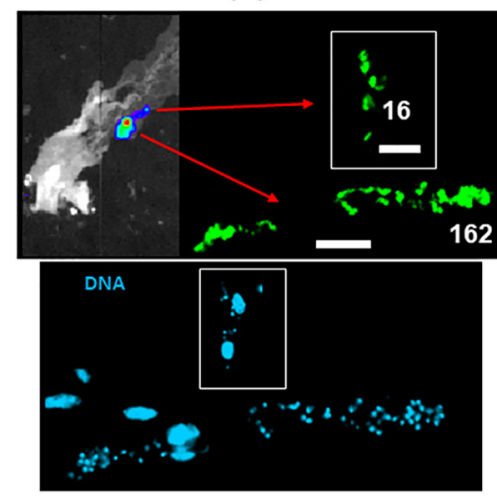

(e)
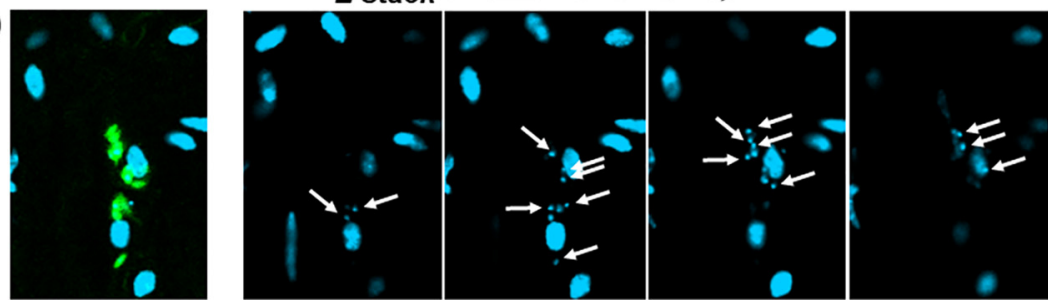

FIG 1 The limit of detection by ex vivo bioluminescence imaging of the murine colon is less than 20 parasites. (a) Ex vivo bioluminescence imaging of a section of the colon from a C3H/HeN mouse chronically infected (155 days postinfection) with $T$. cruzi CL-Luc::Neon (25), pinned luminal side up. The bioluminescence signal is on a linear-scale pseudocolor heat map (same for all bioluminescence images in this figure). (b) Schematic showing the distinct layers of the GI tract (see also Fig. 3a). The dashed red line and arrow indicate the position above which tissue can be peeled off to leave the external colonic wall layers. (c) Bioluminescence image of a colonic wall section after peeling. The insets show the fluorescent parasites (green) detected after exhaustive 3-dimensional imaging of the tissue section (see Materials and Methods) and the numbers detected. Parasites corresponding to one bioluminescent focus (indicated by a question mark) could not subsequently be found, due to technical issues. (d) (Top) External colonic wall layer from a separate mouse showing the correlation of bioluminescence imaging and fluorescence (green), including an infection focus with 16 parasites (left) (bars, $20 \mu \mathrm{m}$ ). (Bottom) Staining with DAPI identifies the location of parasite (small) and host cell (large) DNA. (e) Determination of parasite number. Serial z-sections of the external colonic wall tissue containing the parasite nest shown in panel d indicate how 3-dimensional imaging can be used to calculate the number of parasites on the basis of DNA staining. See Fig. S1 for more detail.

cell remaining relatively low (mouse 1 [M1],6.5; M2,6.7; M3,6.5; M4, 4.6; M5, 19.7; mean $=9.4 ; 95 \%$ confidence interval, $1.15<\mu<16.46$ ) (Fig. 2a, c, and d). In the chronic stage, the situation was different. Of the total parasites in the smooth muscle, more than half were present in mega-nests of $>200$ (Fig. 2c, dashed red line), although most infected cells (>90\%) contained fewer than 50 parasites. Nest size could extend to $>1,000$ parasites. The number per infected cell was determined by z-stacking, which could be done with accuracy even at this level of parasite burden (for details, see Fig. S1 in the supplemental material). In the chronic stage, fully developed trypomastigotes were not apparent in any of the infected cells examined during this study. In contrast, fully developed flagellated trypomastigotes were routinely observed in nests during the acute stage (for an example, see Fig. 2e). We did not find a single mega-nest in external colonic wall tissue derived from any animal during an acute-stage infection, with 63 parasites being the maximum. Both of these findings could indicate higher replication and differentiation rates during the acute stage as the parasite attempts to more rapidly disseminate at the beginning of an infection. As a corollary to this, it may be that a lower growth rate during the chronic stage also benefits the parasite by reducing the immunological footprint of the infection. It will be important to explore this further.

To more accurately determine the specific location of parasites within the colon of chronically infected mice, we made histological sections of paraffin-embedded whole colon tissue derived from both $\mathrm{C} 3 \mathrm{H} / \mathrm{HeN}$ and $\mathrm{BALB} / \mathrm{C}$ mice infected with the $\mathrm{CL}$ Brener dual reporter strain. Using bioluminescence-guided sampling and confocal imaging, we exhaustively searched the tissue sections for fluorescent parasites ( $>100$ sections per mouse). Bioluminescent foci could be well correlated with individual infected host cells 
(a)

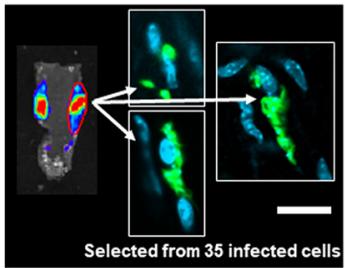

(c)

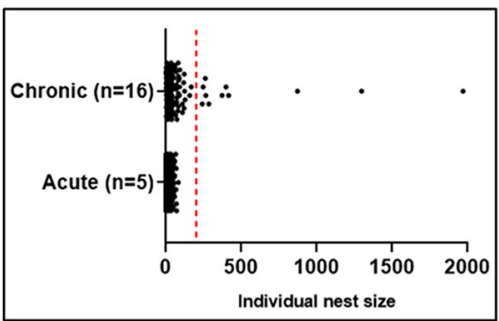

(b)

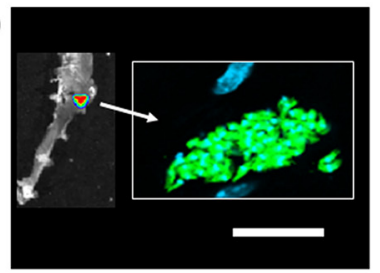

(d)

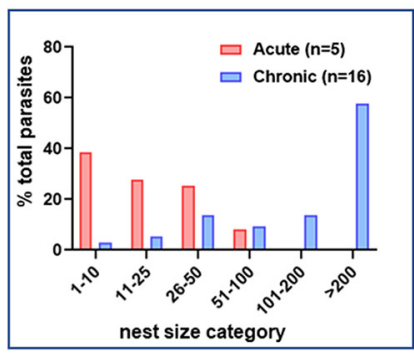

(e)

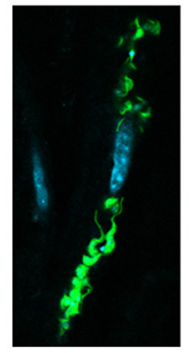

FIG 2 In the external colonic wall of chronic-stage mice, cells infected with more than 200 parasites contain much of the T. cruzi population. (a) Bioluminescence imaging of peeled colon isolated from a $\mathrm{C} 3 \mathrm{H} / \mathrm{HeN}$ mouse 15 days postinfection (acute stage). After mounting, the region of interest (ROI) encompassed by the red line was exhaustively searched by confocal microscopy. Thirty-five infected cells were found within the ROI, 3 of which are shown. Parasites are in green. (b) Using the same approach, the external colonic wall from a chronically infected mouse (183 days postinfection) was assessed. The bioluminescent focus corresponded to a single highly infected host cell. Bars, $20 \mu \mathrm{m}$. (c) Pooled data from T. cruzi-infected cells in peeled colonic wall tissue muscle, isolated from 5 acutely and 16 chronically infected mice. Tissue was examined and the number of parasites per host cell established after the use of z-stacking to provide a 3-dimensional image (Fig. S1). Each dot represents a single infected cell (acute stage, $n=1,198$; chronic stage, $n=140$ ). Dots to the right of the dashed red line indicate infected cells containing $>200$ parasites. (d) The same data set expressed as the percentage of the total parasites detected in the colons of mice in the acute $(n=5)$ and chronic $(n=16)$ stages of infection, by nest size category. (e) An infected cell in the peeled colon of a mouse in the acute stage ( 15 days postinfection) of infection in which the parasites have differentiated to flagellated trypomastigotes.

or small numbers of infected cells in close proximity (Fig. 3b; Fig. S2). Infected cells were most commonly located in the circular muscle layer and only infrequently in the longitudinal muscle, or, despite its larger size and volume, the mucosal layer (Fig. 2; Fig. 3b and c; Fig. S2). No infections of the columnar epithelial cells in the mucosal layer were detected in any mouse. We therefore conclude that in the colon, smooth muscle tissue is the major, although not the exclusive, site of parasite persistence during chronic infection. Consistent with the whole-mount imaging results (Fig. 2c), there was high variability in the number of $T$. cruzi organisms per infected cell in the colonic tissue, ranging from single parasites to nests of $>200$, but no obvious correlation between the parasite burden per cell and the location of the infected cells within the various tissue layers. In the whole-tissue mounts, based on the bioluminescence profile, there was a tendency for the proximal region of the colon to be more highly infected than the middle and distal regions, although this did not reach statistical significance (Wilcoxon rank sum test) (Fig. 4a).

To identify the major cell type(s) which acts as a parasite host during chronic infections of the Gl tract, we single-stained whole mounted external colonic wall sections with specific antibodies against SMA- $\alpha$ (smooth muscle actin- $\alpha$ ), $\beta$-tubulin-3 (a marker for neurons), and CD45 (a broad-range marker of all nucleated hematopoietic cells) (see Materials and Methods). These experiments showed that smooth muscle myocytes were the predominant host cell type (Fig. $4 \mathrm{~b}$ and $\mathrm{c}$ ), with a minority of infected cells stained with the neuronal or leukocyte marker. Interestingly, mega-nests, cells infected with $>200$ parasites, were refractive to staining (Fig. $4 b$ ). In the case of the cytoplasmic markers SMA- $\alpha$ and $\beta$-tubulin-3, this could reflect the fact that their levels are considerably reduced because almost all of the cytoplasm is filled with parasites.

Assessing skeletal muscle and skin as sites of parasite persistence during chronic-stage murine infections. For this study, BALB/c and $\mathrm{C} 3 \mathrm{H} / \mathrm{HeN}$ mice were chronically infected with T. cruzi CL-Luc::Neon (25), and the dissection procedures used 
(a)

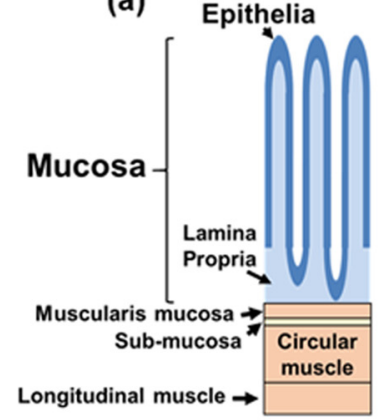

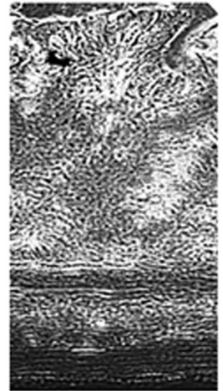

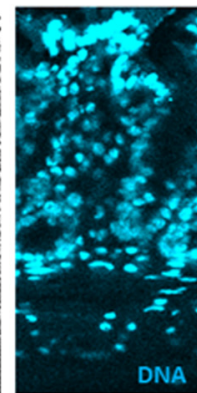

(b)

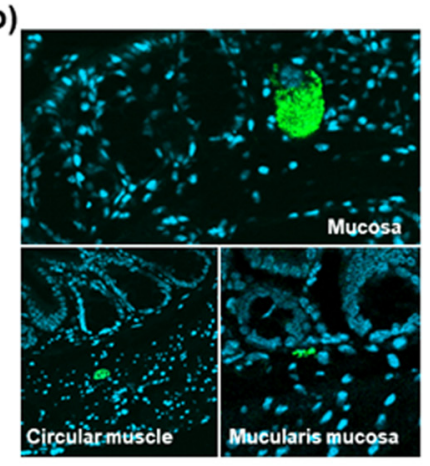

(c)

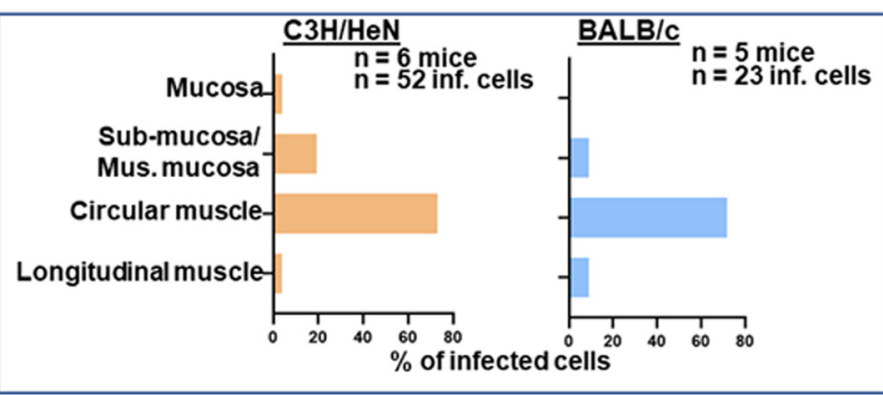

FIG 3 The majority of parasites in the colon of a chronically infected mouse are located in the circular muscle section. (a) Depiction of the layers of the GI tract, correlated with the phase-contrast (left) and stained-DNA (DAPI) (right) images of the same tissue section. (b) Examples of host cells infected with fluorescent parasites (green) detected in different layers of the GI tract (see also Fig. S2). Infection foci were located by confocal imaging of fixed histological sections. (c) Summary of parasite location data obtained using histological sections from chronically infected $\mathrm{C} 3 \mathrm{H} / \mathrm{HeN}$ and $\mathrm{BALB} / \mathrm{c}$ mice.

for ex vivo imaging (Fig. 5a) were further modified to extend the range of tissue sites that could be assessed (see Materials and Methods). Total removal of the skin and fur from the carcass allowed the whole of the skeletal muscle system to be exposed and imaged (Fig. $5 \mathrm{~b}$ and d). The skin could also be placed fur side down and imaged in its entirety after the removal of adipose tissue. All adipose tissue harvested during the dissection process was combined to be imaged separately.

Each $\mathrm{C} 3 \mathrm{H} / \mathrm{HeN}$ mouse registered a bioluminescence signal in the skeletal muscle during chronic-stage infections ( $n=16$ ) (Fig. 5c). It could be inferred from the bioluminescence intensity that the parasite burden in this strain was significantly higher in skeletal muscle than in other organs or tissues, including the $\mathrm{Gl}$ tract and lungs $(P<$ 0.001 , Wilcoxon signed-rank test) (Fig. 5b and c). As previously reported (22), parasite burden and dissemination during chronic stage infections are more extensive in $\mathrm{C} 3 \mathrm{H} / \mathrm{HeN}$ mice than in other mouse models. In line with this, we did not routinely detect highly bioluminescent foci in the skeletal muscle of BALB/c mice (Fig. $5 b$ and c). In addition, levels in the adipose tissue samples of the BALB/c mice were consistently close to background levels, whereas with the $\mathrm{C} 3 \mathrm{H} / \mathrm{HeN}$ mice, more than half displayed a detectable signal ( $>2$ standard deviations [SDs] above background radiance) (Fig. 5c). Following bioluminescence-guided excision (Fig. 5d), infected foci from $\mathrm{C} 3 \mathrm{H} / \mathrm{HeN}$ skeletal muscle were subjected to histological sectioning and examined by confocal microscopy, with parasites detected on the basis of green fluorescence. Consistent with the external colonic wall data (Fig. 1), strong bioluminescent foci corresponded with large mega-nests constituted by many hundreds of parasites (Fig. $5 \mathrm{~d}$ ). Costaining of these skeletal muscle sections with anti-actin- $\alpha$ antibodies revealed that the meganests were internal to the muscle fibers. Therefore, skeletal muscle represents an important site of parasite persistence in chronically infected $\mathrm{C} 3 \mathrm{H} / \mathrm{HeN}$ mice but not in $\mathrm{BALB} / \mathrm{c}$ mice.

Previous studies have shown evidence of $T$. cruzi infection foci localized to skin samples $(20,22)$. However, the extent to which the skin could act as a potential 
(a)
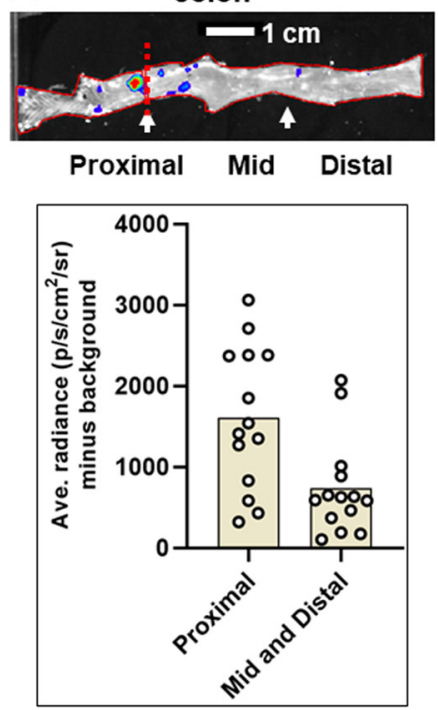

(b)

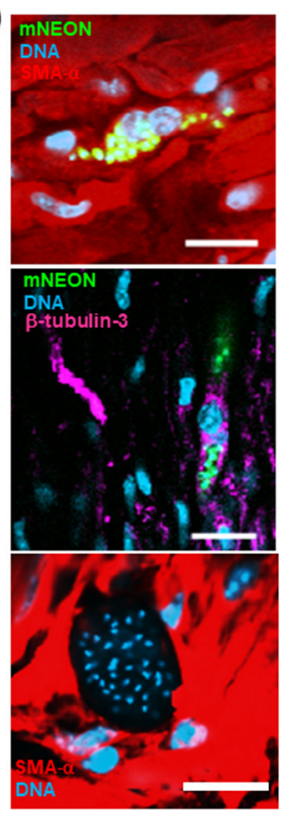

(c)

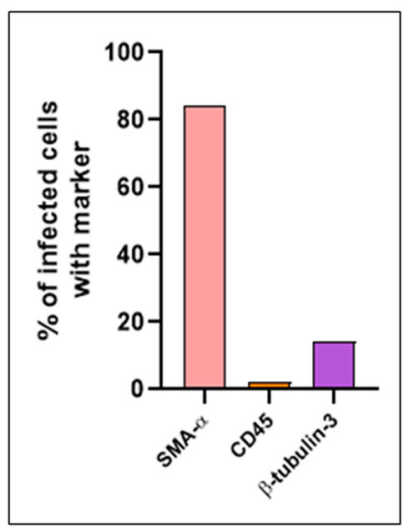

FIG 4 Smooth muscle cells are the predominant infected cell type in the Gl tract of chronically infected mice. (a) Bioluminescence image of the large intestine of a chronically infected $\mathrm{C} 3 \mathrm{H} / \mathrm{HeN}$ mouse indicating the proximal, middle, and distal regions, defined as the first, second, and third segments measured using image J software. Data were analyzed as described in Materials and Methods $(n=14)$ and are presented in the bar chart as the average radiance (photons per second per square centimeter per steradian) minus the background. (b) Illustrative images taken with the mounted external colonic wall section, following staining with cell type-specific antibodies (see Materials and Methods). (Top) Infected smooth muscle cell. (Middle) Infected neuronal cell. (Bottom) A large parasite nest, refractive to staining with any of the 3 markers. (c) Bar chart summarizing distribution of infection by host cell type. External colonic wall sections were singly stained with cell type-specific antibodies: for smooth muscle, SMA- $\alpha$ (4 mice; 24 infected cells, 20 positive); for neuronal cells, $\beta$-tubulin-3 (3 mice; 14 infected cells, 2 positive); for immune cells, CD45 (8 mice; 61 infected cells, 1 positive).

reservoir site has not been evaluated systematically. To investigate this, we infected $\mathrm{C} 3 \mathrm{H} / \mathrm{HeN}$ and BALB/c mice with the bioluminescent $T$. cruzi lines JR (DTU I) and CL Brener (DTU VI) and employed a modified dissection protocol that allowed nearly complete skins from infected mice to be subjected to ex vivo imaging after removal of subcutaneous adipose tissue (see Materials and Methods) (Fig. 6a). Depending on the infection model, between $80 \%$ and $90 \%$ of mice had at least one discernible focus of skin infection (Fig. 6b). For all four parasite-mouse strain combinations, we observed a wide range of skin parasitism patterns, as judged by both the number and the intensity of the bioluminescent foci (Fig. 6a and b). There was some evidence that $\mathrm{C} 3 \mathrm{H} / \mathrm{HeN}$ mice had more $\mathrm{CL}$ Brener skin parasites than BALB/C mice (Fig. $6 \mathrm{~b}$ and c). Infections with the CL Brener strain produced more discrete foci and a higher inferred parasite load than JR infections, although some of this effect could be attributed to lower luciferase expression levels in the JR strain (22). Skin imaging was conducted after removal of subcutaneous adipose tissue by dissection, strongly suggesting that the majority of parasites were resident in the dermis. To visualize parasites at the cellular level, bioluminescence-positive biopsy specimens were processed for thin-section fluorescence imaging from infections with parasites expressing the bioluminescent-fluorescent fusion protein $(\sim 300$ sections from 5 mice). Visualization of infected cells in the skin biopsy specimens was more challenging than for other tissues because of technical difficulties in generating longitudinal sections. Only a single, apparently multinucleated infected cell was identified (Fig. 6d), containing approximately 30 parasites and located within $150 \mu \mathrm{m}$ of the epidermis. Parasites in this anatomical location could have a role in disease transmission. 
(a)

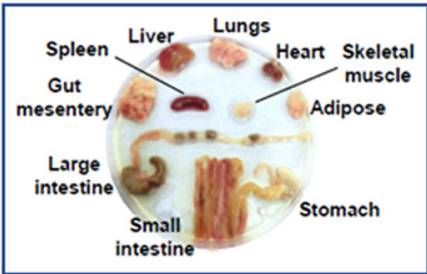

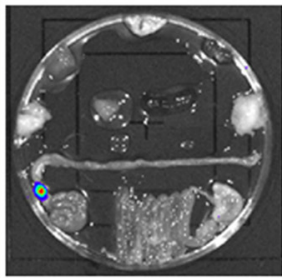

(b)

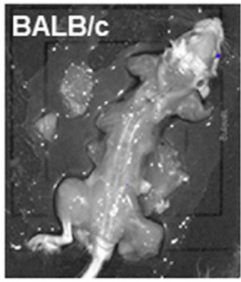

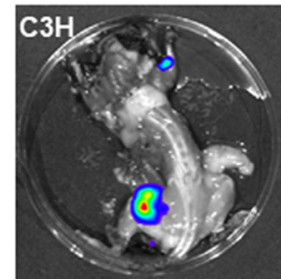

(d)

- $\mathrm{C} 3 \mathrm{H} / \mathrm{HeN}(\mathrm{n}=15)$

- BALB/c (n=7)

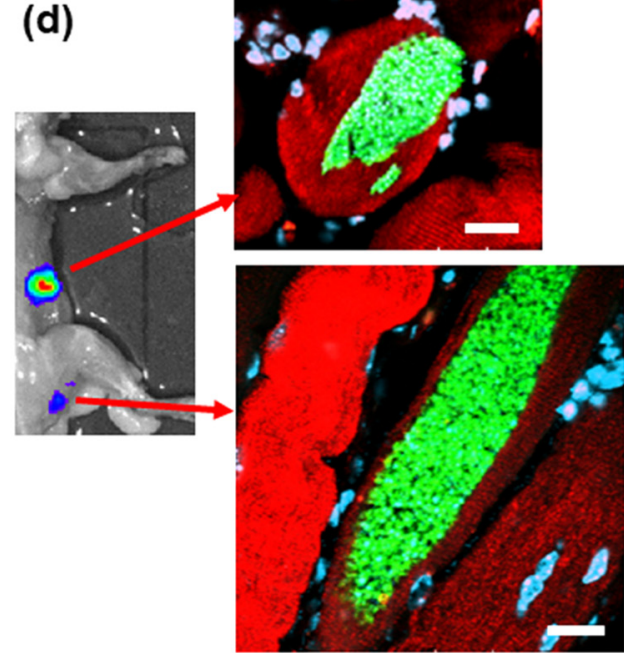

FIG 5 Skeletal muscle is a major site of parasite persistence during chronic T. cruzi infections in C3H/HeN mice but not BALB/c mice. (a) Ex vivo imaging of organs and tissues from a BALB/c mouse chronically infected with bioluminescent $T$. cruzi CL Brener. (b) Dorsal bioluminescence imaging of chronically infected $\mathrm{BALB} / \mathrm{C}$ and $\mathrm{C} 3 \mathrm{H} / \mathrm{HeN}$ mice following removal of internal organs, fur, skin, and major adipose depots (see Materials and Methods). (c) Fold change in radiance (photons per second per square centimeter per steradian) established by ex vivo bioluminescence imaging of internal tissues and organs and skeletal muscle as imaged in panels a and b. The dashed line indicates the detection threshold, equal to the mean plus 2 SDs of the bioluminescence background derived from corresponding empty regions of interest in tissue from age-matched uninfected mice. For technical reasons, on a small number of occasions, data could not be acquired for tissue samples from some mice (e.g., adipose tissue). (d) Bioluminescent foci from skeletal muscle were excised, and histological sections were prepared and then scanned by confocal microscopy (see Materials and Methods). Sections were stained with specific markers for muscle (red, actin- $\alpha$ ) and DNA (blue/turquoise, DAPI). Parasites can be identified by green fluorescence. Bars, $20 \mu \mathrm{m}$.

\section{DISCUSSION}

Chronic Chagas disease in humans is characterized by long-term parasite persistence at levels that are difficult to monitor with accuracy, even using highly sensitive PCR-based techniques. This has been a complicating factor in diagnosis and in monitoring therapeutic cure during clinical trials $(29,30)$. Additionally, it has not been possible to identify the main tissues and/or organs that function as sites of parasite persistence in an immunological environment that otherwise tightly controls the infection. Information on the systemic parasite load and location throughout the infection would provide a better understanding of disease progression and the determinants of the wide spectrum of symptoms that are characteristic of this chronic condition. Experimental animal models have proved to be invaluable experimental tools for providing data in these areas, particularly in combination with genetically modified parasite reporter strains. These systems can provide real-time readouts on infection dynamics $(20,22,31)$, insights into tissue tropism (26), and information on the influence of host and parasite genetics. The murine models used in the present study display an infection profile similar to that in humans, have proved to be predictive of drug efficacy, and display a spectrum of cardiac pathology that mirrors aspects of the human disease.

Here, we exploited parasites that express fusion proteins containing bioluminescent and fluorescent domains. Together with improved tissue preparation techniques, this has enabled us to achieve a limit of detection by ex vivo imaging that is less than 20 
(a)

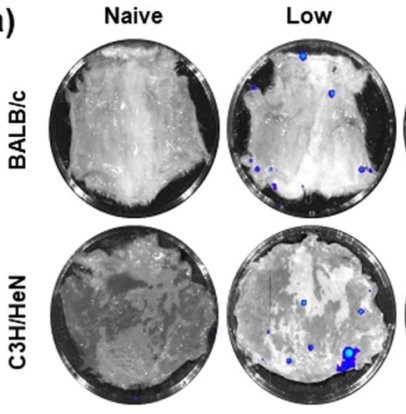

(b)

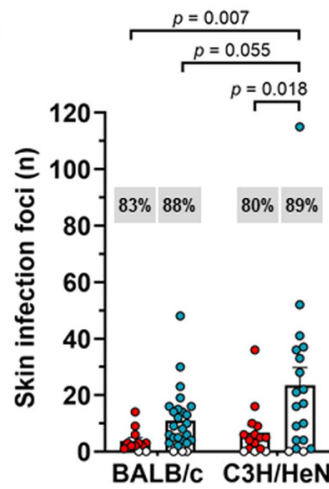

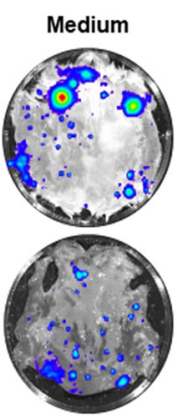

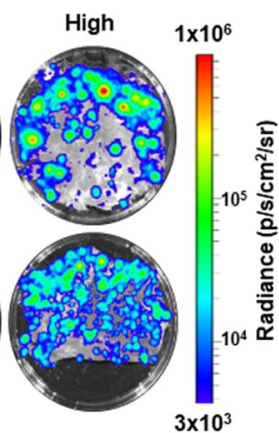

(c) (d)

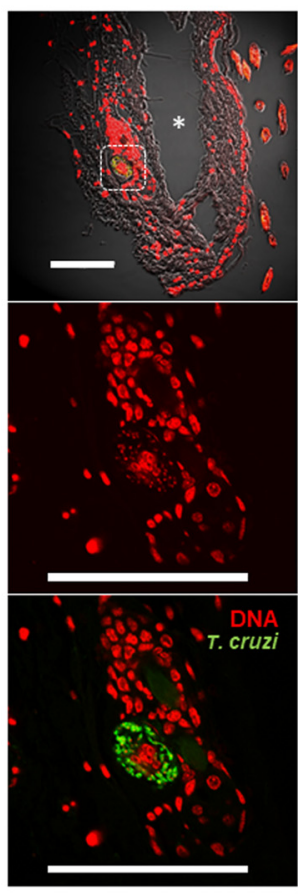

FIG 6 The skin is a major site of parasite persistence during chronic $T$. cruzi infections in mice. (a) Ex vivo bioluminescence imaging of skin (adipose tissue removed) from chronically infected BALB/c and $\mathrm{C} 3 \mathrm{H} / \mathrm{HeN}$ mice ( $>150$ days postinfection) showing representative examples of low, medium, and high parasite loads. The bioluminescence signal is on a $\log _{10}$ scale pseudocolor heat map. (b and c) Quantification of the number of discrete infection foci (b) and the bioluminescence intensity (c) for each skin. Data points represent individual animals, with empty circles indicating skins having zero radiance above background. Mean values and standard errors of the mean (SEM) are shown. Percentages in gray boxes (b) are the percentages of animals with at least one focus above the bioluminescence threshold. Infections with both T. cruzi CL Brener and JR bioluminescent strains were assessed (12 to 26 animals per combination, 3 to 4 independent experiments). Groups were compared by 2-way ANOVA. (d) Confocal micrographs showing fluorescent $C L$ Brener parasites in an infected cell within the dermis of a BALB/C mouse 230 days postinfection (surface to the right). The asterisk indicates a gap resulting from a cutting artifact. The bottom two images highlight the region in the white boxed area in the top image. Bars, $100 \mu \mathrm{m}$. Magnifications, $\times 200$ (top) and $\times 630$ (middle and bottom).

parasites (Fig. $2 \mathrm{~d}$ and e). By facilitating the routine detection of parasites in their tissue context, at the level of individual host cells, these approaches have overcome a major barrier that has restricted progress in the investigation of chronic $T$. cruzi infections. Previous studies using bioluminescent parasites identified the $\mathrm{Gl}$ tract as a major site of parasite persistence during the chronic stage $(20,22)$. However, these studies, which involved several mouse-parasite strain combinations, revealed few details on the nature of host cells or on their precise location within tissue. We have now shown that in the colon, the circular smooth muscle coat is the predominant site of parasite persistence (Fig. 3) and that smooth muscle myocytes are the main infected host cell type. Enteric neurons can also be parasitized, but such infections are much less common (Fig. 4). The extent to which this apparent tropism is determined by a metabolic preference for the corresponding regions or cells or by the immunological microenvironment is not known. Interestingly, external colonic wall-resident $\mathrm{CD} 45^{+}$hematopoietic cells were rarely infected (Fig. 4a), even though myeloid cells are well-known targets during acute-stage infection in other sites, such as the spleen and bone marrow. We also failed to find a single instance where parasites infected epithelial cells on the mucosal surface, suggesting that parasitized cells or trypomastigotes are unlikely to be shed into the lumen of the large intestine.

Experiments have shown that parasite survival in the colon during chronic infections reflects crucial differences between the immune environment of certain $\mathrm{Gl}$ tract regions and systemic sites (22). Immunosuppression of infected mice leads to widespread parasite dissemination to other less permissive organs and tissues, including the heart. 
There is clearly a host genetic component to this immune restriction, since the same parasite strains display a wider tissue distribution in $\mathrm{C} 3 \mathrm{H} / \mathrm{HeN}$ mice than in the $\mathrm{BALB} / \mathrm{C}$ strain (Fig. $5 \mathrm{c}$ ), a phenomenon which is associated with increased cardiac pathology (22). In the human population, this highlights the idea that genetic diversity affecting the functioning of the immune system and its ability to restrict the tissue range of $T$. cruzi to reservoir sites could be a major determinant of Chagas disease pathology. Within $\mathrm{C} 3 \mathrm{H} / \mathrm{HeN}$ mice, skeletal muscle was also found to be an important site of persistence during the chronic stage, whereas in the BALB/c strain, parasites were far less evident in this location (Fig. 5c). Some T. cruzi strains have been reported to be myotropic in mice, with pathological outcomes that include paralyzing myositis and skeletal muscle vasculitis (32). Myocyte infections could also provide the parasite with access to myoglobin, a source of heme or iron that may contribute to a nutritional environment that is favorable for replication. The ability of high numbers of parasites to survive long-term in the skeletal muscle, compared to other sites, indicates that this tissue can function as a more immunologically permissive niche in the genetic background of the $\mathrm{C} 3 \mathrm{H} / \mathrm{HeN}$ mouse. Strikingly, myocytes in this tissue could contain several hundred parasites (Fig. 5d). We have previously suggested that the existence of large mega-nests such as these could have implications for drug efficacy (26), with parasites in the center of the nest having reduced drug exposure compared to those on the periphery, possibly contributing to treatment failure. This form of "herd protection" may not be captured in the type of high-throughput in vitro screening assays that are a common feature of the drug development process. It will also be interesting to explore whether some parasites within these mega-nests adopt a metabolically quiescent state, analogous to the dormant phenotype recently reported (14).

Our study has also demonstrated that the skin is another location where T. cruzi can frequently be detected during chronic infections. In both $\mathrm{C} 3 \mathrm{H} / \mathrm{HeN}$ and $\mathrm{BALB} / \mathrm{c}$ mice, infection levels of $>80 \%$ were observed, although there was considerable variability in the level of infectivity in terms of the number of bioluminescent foci and the total parasite load. The extent of this became apparent only when the entire skin of the mouse was examined by ex vivo imaging with the fur side down (Fig. 6), presumably because bioluminescent signals at the levels displayed by the majority of foci are masked by the fur when monitored by in vivo imaging. Skin-localized parasites are a common and well-characterized feature of many Leishmania species infections. More recently, it has also been reported that Trypanosoma brucei can also be detected in the skin of both mice and humans and that these parasites could have important roles in persistence and transmission $(33,34)$. Until now, descriptions of cutaneous T. cruzi have been restricted to intermittent (chagoma and Romaña's sign) or atypical manifestations of the acute stage (35) or to reactivation of chronic infections as a result of immunosuppression $(36,37)$. Parasites in the dermal layers (Fig. 6) have the potential to play a crucial role in transmission of Chagas disease, since they would have ready access to the triatomine vector during a blood meal. The extent to which $T$. cruzi parasites are localized to the skin during human chronic infections will also be of interest, since this could impact transmission dynamics, as has been suggested from a detailed spatial analysis of Leishmania donovani in the skin of infected mice (38). It will also be important to determine whether these skin-resident parasites are persistent at this location or whether they represent a transient population that is constantly reseeded from other permissive niches, such as the Gl tract (13). Resolution of this question will help to inform drug design by revealing whether the ability to access parasites in the dermal layers has to be a prerequisite property of novel therapeutics. In murine models of $T$. brucei infection, adipose tissue also forms an important parasite reservoir (39). This was not the case with chronic T. cruzi infections of BALB/C mice (Fig. 5c), where parasites were largely absent from these tissue sites. Bioluminescent foci were detected in the adipose tissues in approximately half of the chronically infected $\mathrm{C} 3 \mathrm{H} / \mathrm{HeN}$ mice. However, rather than a specific tropism, this may simply reflect the immunological context in $\mathrm{C} 3 \mathrm{H} / \mathrm{HeN}$ mice, which allows more extensive parasite distribution than in other mouse models (22). 
In summary, we have provided new data on the sites of parasite persistence in murine models of chronic Chagas disease. This provides a framework for identifying the immunological parameters that determine whether a specific tissue site can act as a permissive niche and for investigating the extent to which the parasite itself has a direct role in the process.

\section{MATERIALS AND METHODS}

Ethics. Animal work was carried out under UK Home Office project licenses (PPL 70/8207 and P9AEE04E4) and approved by the LSHTM Animal Welfare and Ethical Review Board. Experiments were conducted in accordance with the UK Animals (Scientific Procedures) Act 1986.

Parasites, mice, and infections. Two parasite reporter strains were used; the bioluminescent and fluorescent $T$. cruzi CL-Luc::Neon, a CL Brener clone (DTU VI) which expresses a fusion protein containing red-shifted luciferase linked to mNeonGreen $(20,25)$, and a JR clone (DTU I) which expresses red-shifted luciferase $(19,22)$. Epimastigotes were grown at $28^{\circ} \mathrm{C}$ in RPMI 1640 supplemented with $0.5 \%$ (wt/vol) tryptone, $20 \mathrm{mM}$ HEPES (pH 7.2), $30 \mathrm{mM}$ hemin, 10\% heat-inactivated fetal bovine serum (FBS), $2 \mathrm{mM}$ sodium glutamate, $2 \mathrm{mM}$ sodium pyruvate, $100 \mu \mathrm{g} / \mathrm{ml}$ streptomycin, and $100 \mathrm{U} / \mathrm{ml}$ penicillin, with $150 \mu \mathrm{g} / \mathrm{ml}$ hygromycin (CL Brener) or $100 \mu \mathrm{g} / \mathrm{ml} \mathrm{G} 418$ (JR) as selective drugs. Metacyclic trypomastigotes (MTs) were obtained by transfer to Grace's insect transformation medium (40). MTs were harvested after 4 to 7 days, when 70 to $90 \%$ of parasites had differentiated. Tissue culture trypomastigotes were obtained from infected MA104 cells grown at $37^{\circ} \mathrm{C}$ using minimal Eagle medium supplemented with $10 \%$ heat-inactivated FBS.

$\mathrm{BALB} / \mathrm{C}$ and $\mathrm{C} 3 \mathrm{H} / \mathrm{HeN}$ mice were purchased from Charles River (United Kingdom), and CB17 SCID mice were bred in-house. Animals were maintained under pathogen-free conditions in individually ventilated cages. They experienced a 12-h light-dark cycle and had access to food and water ad libitum. Female mice aged 8 to 12 weeks were used. SCID mice were infected with $1 \times 10^{4}$ in vitro-derived tissue culture trypomastigotes in $0.2 \mathrm{ml}$ phosphate-buffered saline (PBS) via intraperitoneal (i.p.) injection. Other mice were infected by i.p injection with $1 \times 10^{3}$ bloodstream trypomastigotes derived from blood of parasitemic SCID mice. Infection by different routes (intravenous, subcutaneous, or oral) does not result in different parasite distribution profiles in tissues or organs $(20,31)$. All SCID mice developed fulminant infections and were euthanized at, or before, humane endpoints by lethal injection with 0.1 to $0.2 \mathrm{ml}$ Dolethal.

Ex vivo bioluminescence imaging. For ex vivo imaging, mice were injected with $150 \mathrm{mg} / \mathrm{kg}$ D-luciferin i.p. and then sacrificed by lethal i.p. injection 5 min later $(20,21)$. Mice were perfused with $10 \mathrm{ml}$ of $0.3 \mathrm{mg} / \mathrm{ml} \mathrm{D}$-luciferin in PBS via the heart. Organs and tissues were imaged using the IVIS Spectrum system (Caliper Life Science) and Livinglmage 4.7.2 software. First, the heart, lungs, spleen, liver, Gl tract, GI mesenteric tissue, kidneys, and all visceral adipose tissue were transferred to a petri dish in a standardized arrangement, soaked in $0.3 \mathrm{mg} / \mathrm{ml}$ D-luciferin in PBS, and imaged using maximum detection settings (2-min exposure, large binning). Then, the skin was removed from the carcass, and subcutaneous adipose tissue was recovered (41) and added to the visceral fat, creating a whole adipose tissue sample, which was imaged in the same way. The skin was placed fur down, soaked in $0.3 \mathrm{mg} / \mathrm{ml}$ D-luciferin, and imaged under the same conditions as the internal organs. The skeletal muscle was placed dorsal side up, soaked in $0.3 \mathrm{mg} / \mathrm{ml}$ D-luciferin, and imaged as described above.

To assess infection intensities in ex vivo tissues, regions of interest (ROI) were drawn to quantify bioluminescence expressed as radiance (photons per second per square centimeter per steradian). Because different tissue types have different background radiances, we normalized data from infected mice using matching tissues from uninfected controls $(n=4)$ and used the fold change in radiance, compared with the tissue-specific controls, as the final measure. Detection thresholds for ex vivo imaging were determined using the fold change in radiance for $\mathrm{ROI}$ from infected mice compared with matching empty ROI in control mice of comparable age.

In some experiments, the colon was removed after standard imaging, an incision was made down the line of mesentery attachment, and the tissue was pinned out under a dissection microscope. Using ultrafine tweezers, large sections of the smooth muscular coat from the other layers were peeled off, while the tissue remained bathed in $0.3 \mathrm{mg} / \mathrm{ml}$ D-luciferin (41). After imaging, luciferin was removed by two washes with PBS. Tissue was fixed with $4 \%$ paraformaldehyde for $45 \mathrm{~min}$, followed by two washes with PBS (41). External colonic wall tissue was then mounted whole in Vectashield antifade mounting medium with 4',6-diamidino-2-phenylindole (DAPI; Vector Laboratories) and imaged as described below.

Histological sections were created after bioluminescence-guided excision of infection foci from skeletal muscle and colon tissue $(25,26,41)$. Biopsy specimens were first incubated in $95 \% \mathrm{EtOH}$ at $4^{\circ} \mathrm{C}$ overnight and then washed in $100 \% \mathrm{EtOH}$ (four times, $10 \mathrm{~min}$ each), followed by xylene (twice, $12 \mathrm{~min}$ each). Samples were embedded by placing in melted paraffin wax (twice, $12 \mathrm{~min}$ each). The wax was allowed to set, and the embedded pieces were sectioned into 5- to 20- $\mu \mathrm{m}$ histological sections using a microtome. The sections were melted, the paraffin was dissolved in xylene for $30 \mathrm{~s}$, and then sections were washed in $95 \%$ ethanol (three times, 1 min each), followed by 3 washes in PBS. Sections were mounted in Vectashield and imaged using the Zeiss LSM880 confocal microscope. For precise counting of intracellular parasites, samples were imaged in 3 dimensions, with the appropriate scan zoom setting, and the files were analyzed using Image J software (Fig. S1).

Antibody staining. Deparaffinized sections were incubated at $4^{\circ} \mathrm{C}$ overnight in primary antibody diluted at 1:200 in PBS- 0.5\% FBS. Antibodies against $\beta$-tubulin-3 (catalog no. 802001; BioLegend), CD45 (catalog no. 70-0451; Tonbo Biosciences), smooth muscle actin (catalog no. A2547; Sigma), and skeletal 
muscle actin (catalog no. MA5-12542; Thermo Fisher) were used to stain for neuronal, nucleated hematopoietic, smooth muscle, and skeletal muscle cells, respectively. Secondary antibodies (Thermo Fisher) diluted 1:500 in PBS were incubated on sections for $3 \mathrm{~h}$ at room temperature before mounting. Both primary and secondary antibodies were removed by three 2-min washes in PBS. For staining of whole colon external wall sections, the tissue was submerged in the primary antibody dilution for $48 \mathrm{~h}$ at $4^{\circ} \mathrm{C}$ and then submerged in the secondary dilution at room temperature for $3 \mathrm{~h}$ before three 2-min washes in PBS.

Statistics. The Shapiro-Wilk test for normality and the Wilcoxon rank sum nonparametric test were used to analyze the data presented in Fig. 4 and 5. Two-way analysis of variance (ANOVA) with Tukey's post hoc correction test was used for Fig. 6. All tests were performed in GraphPad Prism v.8.

\section{SUPPLEMENTAL MATERIAL}

Supplemental material is available online only.

FIG S1, TIF file, $0.3 \mathrm{MB}$.

FIG S2, TIF file, $0.7 \mathrm{MB}$.

\section{ACKNOWLEDGMENTS}

This work was supported by UK Medical Research Council (MRC) grants MR/ T015969/1 to J.M.K. and MR/R021430/1 to M.D.L. and by MRC LID (DTP) studentship MR/N013638/1 to A.I.W. The funders had no role in study design, data collection and interpretation, or the decision to submit the work for publication.

\section{REFERENCES}

1. WHO. 2020. Chagas disease. www.who.int/news-room/fact-sheets/detail/ chagas-disease-(american-trypanosomiasis).

2. Requena-Mendez A, Aldasoro E, de Lazzari E, Sicuri E, Brown M, Moore DA, Gascon J, Muñoz J. 2015. Prevalence of Chagas disease in LatinAmerican migrants living in Europe: a systematic review and metaanalysis. PLoS Negl Trop Dis 9:e0003540. https://doi.org/10.1371/journal .pntd.0003540.

3. Bern C, Messenger LA, Whitman JD, Maguire JH. 2019. Chagas disease in the United States: a public health approach. Clin Microbiol Rev 33: e00023-19. https://doi.org/10.1128/CMR.00023-19.

4. Cardillo F, de Pinho RT, Antas PR, Mengel J. 2015. Immunity and immune modulation in Trypanosoma cruzi infection. Pathog Dis 73:ftv082. https:// doi.org/10.1093/femspd/ftv082.

5. Tarleton RL. 2015. CD8+ T cells in Trypanosoma cruzi infection. Semin Immunopathol 37:233-238. https://doi.org/10.1007/s00281-015-0481-9.

6. Ribeiro AL, Nunes MP, Teixeira MM, Rocha MO. 2012. Diagnosis and management of Chagas disease and cardiomyopathy. Nat Rev Cardiol 9:576-589. https://doi.org/10.1038/nrcardio.2012.109.

7. Cunha-Neto E, Chevillard C. 2014. Chagas disease cardiomyopathy: immunopathology and genetics. Mediators Inflamm 2014:683230. https:// doi.org/10.1155/2014/683230.

8. Wilkinson SR, Kelly JM. 2009. Trypanocidal drugs: mechanisms, resistance and new targets. Exp Rev Mol Med 11:e31. 1-24. https://doi.org/ 10.1017/S1462399409001252.

9. Gaspar L, Moraes CB, Freitas-Junior LH, Ferrari S, Costantino L, Costi MP, Coron RP, Smith TK, Siqueira-Neto JL, McKerrow JH, Cordeiro-da-Silva A. 2015. Current and future chemotherapy for Chagas disease. Curr Med Chem 22:4293-4312. https://doi.org/10.2174/0929867322666151015120804.

10. Katsuno K, Burrows JN, Duncan K, Hooft van Huijsduijnen R, Kaneko T, Kita K, Mowbray CE, Schmatz D, Warner P, Slingsby BT. 2015. Hit and lead criteria in drug discovery for infectious diseases of the developing world. Nat Rev Drug Discov 14:751-758. https://doi.org/10.1038/nrd4683.

11. Chatelain E. 2017. Chagas disease research and development: is there light at the end of the tunnel? Comput Struct Biotechnol J 15:98-103. https://doi.org/10.1016/j.csbj.2016.12.002.

12. Pack AD, Collins MH, Rosenberg CS, Tarleton RL. 2018. Highly competent, non-exhausted CD8 + T cells continue to tightly control pathogen load throughout chronic Trypanosoma cruzi infection. PLoS Pathog 14: e1007410. https://doi.org/10.1371/journal.ppat.1007410.

13. Lewis MD, Kelly JM. 2016. Putting Trypanosoma cruzi dynamics at the heart of Chagas disease. Trends Parasitol 32:899-911. https://doi.org/10 .1016/j.pt.2016.08.009.

14. Sánchez-Valdéz FJ, Padilla A, Wang W, Orr D, Tarleton RL. 2018. Spontaneous dormancy protects Trypanosoma cruzi during extended drug exposure. Elife 7:e34039. https://doi.org/10.7554/eLife.34039.
15. Duarte JG, Nascimento RD, Martins PR, d'Ávila Reis D. 2017. An autopsybased study of Trypanosoma cruzi persistence in organs of chronic chagasic patients and its relevance for transplantation. Transpl Infect Dis 19:e12783. https://doi.org/10.1111/tid.12783.

16. Hyland KV, Asfaw SH, Olson CL, Daniels MD, Engman DM. 2008. Bioluminescent imaging of Trypanosoma cruzi infection. Int J Parasitol 38: 1391-1400. https://doi.org/10.1016/j.jpara.2008.04.002.

17. Silberstein E, Serna C, Fragoso SP, Nagarkatti R, Debrabant A. 2018. A novel nanoluciferase-based system to monitor Trypanosoma cruzi infection in mice by bioluminescence imaging. PLoS One 13:e0195879. https:// doi.org/10.1371/journal.pone.0195879.

18. Calvet CM, Choi JY, Thomas D, Suzuki B, Hirata K, Lostracco-Johnson S, de Mesquita LB, Nogueira A, Meuser-Batista M, Silva TA, Siqueira-Neto JL, Roush WR, de Souza Pereira MC, McKerrow JH, Podust LM. 2017. 4-Aminopyridyl-based lead compounds targeting CYP51 prevent spontaneous parasite relapse in a chronic model and improve cardiac pathology in an acute model of Trypanosoma cruzi infection. PLoS Negl Trop Dis 11:e0006132. https://doi.org/10.1371/journal.pntd.0006132.

19. Branchini BR, Ablamsky DM, Davis AL, Southworth TL, Butler B, Fan F, Jathoul AP, Pule MA. 2010. Red-emitting luciferases for bioluminescence reporter and imaging applications. Anal Biochem 396:290-297. https:// doi.org/10.1016/j.ab.2009.09.009.

20. Lewis MD, Fortes Francisco A, Taylor MC, Burrell-Saward H, McLatchie AP, Miles MA, Kelly JM. 2014. Bioluminescence imaging of chronic Trypanosoma cruzi infections reveals tissue-specific parasite dynamics and heart disease in the absence of locally persistent infection. Cell Microbiol 16:1285-1300. https://doi.org/10.1111/cmi.12297.

21. Lewis MD, Fortes Francisco A, Taylor MC, Kelly JM. 2015. A new experimental model for assessing drug efficacy against Trypanosoma cruzi infection based on highly sensitive in vivo imaging. J Biomol Screen 20:36-43. https://doi.org/10.1177/1087057114552623.

22. Lewis MD, Fortes Francisco A, Taylor MC, Jayawardhana S, Kelly JM. 2016. Host and parasite genetics shape a link between Trypanosoma cruzi infection dynamics and chronic cardiomyopathy. Cell Microbiol 18: 1429-1443. https://doi.org/10.1111/cmi.12584.

23. Francisco AF, Lewis MD, Jayawardhana S, Taylor MC, Chatelain E, Kelly JM. 2015. The limited ability of posaconazole to cure both acute and chronic Trypanosoma cruzi infections revealed by highly sensitive in vivo imaging. Antimicrob Agents Chemother 59:4653-4661. https://doi.org/ 10.1128/AAC.00520-15.

24. Francisco AF, Jayawardhana S, Taylor MC, Lewis MD, Kelly JM. 2018. Assessing the effectiveness of curative benznidazole treatment in preventing chronic cardiac pathology in experimental models of Chagas disease. Antimicrob Agents Chemother 62:e00832-18. https://doi.org/10 .1128/AAC.00832-18. 
25. Costa FC, Francisco AF, Jayawardhana S, Calderano SG, Lewis MD, OImo F, Beneke T, Gluenz E, Sunter J, Dean S, Kelly JM, Taylor MC. 2018. Expanding the toolbox for Trypanosoma cruzi: a parasite line incorporating a bioluminescence-fluorescence dual reporter and streamlined CRISPR/Cas9 functionality for rapid in vivo localisation and phenotyping. PLoS Negl Trop Dis 12:e0006388. https://doi.org/10.1371/journal.pntd .0006388 .

26. Taylor MC, Ward A, Olmo F, Jayawardhana S, Francisco AF, Lewis MD Kelly JM. 2020. Intracellular DNA replication and differentiation of Trypanosoma cruzi is asynchronous within individual host cells in vivo at all stages of infection. PLoS Negl Trop Dis 14:e0008007. https://doi.org/ 10.1371/journal.pntd.0008007.

27. Francisco AF, Jayawardhana $S$, Lewis MD, White $K L$, Shackleford $D M$, Chen G, Saunders J, Osuna-Cabello M, Read KD, Charman SA, Chatelain E, Kelly JM. 2016. Nitroheterocyclic drugs cure experimental Trypanosoma cruzi infections more effectively in the chronic stage than in the acute stage. Sci Rep 6:35351. https://doi.org/10.1038/srep35351.

28. Mann GS, Francisco AF, Jayawardhana S, Taylor MC, Lewis MD, Olmo F, López-Camacho C, Oliveira de Freitas E, Leoratti FMS, Reyes-Sandoval A, Kelly JM. 2020. Drug-cured experimental Trypanosoma cruzi infections confer long-lasting and cross-strain protection. PLoS Negl Trop Dis 14:e0007717. https://doi.org/10.1371/journal.pntd.0007717.

29. Molina I, Gómez i Prat J, Salvador F, Treviño B, Sulleiro E, Serre N, Pou D, Roure S, Cabezos J, Valerio L, Blanco-Grau A, Sánchez-Montalvá A, Vidal X, Pahissa A. 2014. Randomized trial of posaconazole and benznidazole for chronic Chagas disease. N Engl J Med 370:1899-1908. https://doi .org/10.1056/NEJMoa1313122.

30. Morillo CA, Marin-Neto JA, Avezum A, Sosa-Estani S, Rassi A, Jr, Rosas F, Villena E, Quiroz R, Bonilla R, Britto C, Guhl F, Velazquez E, Bonilla L, Meeks B, Rao-Melacini P, Pogue J, Mattos A, Lazdins J, Rassi A, Connolly SJ, Yusuf S, BENEFIT Investigators. 2015. Randomized trial of benznidazole for chronic Chagas' cardiomyopathy. N Engl J Med 373:1295-1306. https://doi.org/10.1056/NEJMoa1507574.

31. Lewis MD, Francisco AF, Jayawardhana S, Langston H, Taylor MC, Kelly JM. 2018. Imaging the development of chronic Chagas disease after oral transmission. Sci Rep 8:11292. https://doi.org/10.1038/s41598-018 -29564-7.

32. Weaver JD, Hoffman VJ, Roffe E, Murphy PM. 2019. Low-level parasite persistence drives vasculitis and myositis in skeletal muscle of mice chronically infected with Trypanosoma cruzi. Infect Immun 87:e00081-19. https://doi.org/10.1128/IAI.00081-19.
33. Capewell $P$, Cren-Travaillé $C$, Marchesi $F$, Johnston $P$, Clucas $C$, Benson RA, Gorman TA, Calvo-Alvarez E, Crouzols A, Jouvion G, Jamonneau V, Weir W, Stevenson ML, O'Neill K, Cooper A, Swar NK, Bucheton B, Ngoyi DM, Garside P, Rotureau B, MacLeod A. 2016. The skin is a significant but overlooked anatomical reservoir for vector-borne African trypanosomes. Elife 5:e17716. https://doi.org/10.7554/eLife.17716.

34. Caljon G, Van Reet N, De Trez C, Vermeersch M, Pérez-Morga D, Van Den Abbeele J. 2016. The dermis as a delivery site of Trypanosoma brucei for tsetse flies. PLoS Pathog 12:e1005744. https://doi.org/10.1371/journal .ppat. 1005744.

35. Hemmige V, Tanowitz H, Sethi A. 2012. Trypanosoma cruzi infection: a review with emphasis on cutaneous manifestations. Int J Dermatol 51:501-508. https://doi.org/10.1111/j.1365-4632.2011.05380.x.

36. Gallerano V, Consigli J, Pereyra S, Gómez Zanni S, Danielo C, Gallerano $\mathrm{RH}$, Guidi A. 2007. Chagas' disease reactivation with skin symptoms in a patient with kidney transplant. Int J Dermatol 46:607-610. https://doi .org/10.1111/j.1365-4632.2007.03127.x.

37. Riganti J, Guzzi Maqueda M, Baztan Piñero MC, Volonteri VI, Galimberti RL. 2012. Reactivation of Chagas' disease: cutaneous manifestations in two immunosuppressed patients. Int J Dermatol 51:829-834. https:// doi.org/10.1111/j.1365-4632.2011.05224.x.

38. Doehl JSP, Bright Z, Dey S, Davies H, Magson J, Brown N, Romano A, Dalton JE, Pinto Al, Pitchford JW, Kaye PM. 2017. Skin parasite landscape determines host infectiousness in visceral leishmaniasis. Nat Commun 8:57. https://doi.org/10.1038/s41467-017-00103-8.

39. Trindade S, Rijo-Ferreira F, Carvalho T, Pinto-Neves D, Guegan F, ArestaBranco F, Bento F, Young SA, Pinto A, Van Den Abbeele J, Ribeiro RM, Dias S, Smith TK, Figueiredo LM. 2016. Trypanosoma brucei parasites occupy and functionally adapt to the adipose tissue in mice. Cell Host Microbe 19:837-848. https://doi.org/10.1016/j.chom.2016.05.002.

40. Isola EL, Lammel EM, González Cappa SM. 1986. Trypanosoma cruzi: differentiation after interaction of epimastigotes and Triatoma infestans intestinal homogenate. Exp Parasitol 62:329-335. https://doi.org/10 .1016/0014-4894(86)90039-1.

41. Taylor MC, Francisco AF, Jayawardhana S, Mann GS, Ward Al, Olmo F, Lewis MD, Kelly JM. 2019. Exploiting genetically modified dual-reporter strains to monitor experimental Trypanosoma cruzi Infections and hostparasite interactions. Methods Mol Biol 1955:147-163. https://doi.org/ 10.1007/978-1-4939-9148-8_11. 